\section{Relação do índice de massa corporal, da relação cintura-quadril e da circunferência abdominal com a mortalidade em mulheres idosas: seguimento de 5 anos}

\author{
Relationship between body mass index, waist \\ circumference, and waist-to-hip ratio and mortality \\ in elderly women: a 5-year follow-up study
}

\author{
1 Departamento de Clínica \\ Médica, Universidade \\ Estadual de Londrina, \\ Londrina, Brasil. \\ 2 Faculdade de Medicina, \\ Universidade de São Paulo, \\ São Paulo, Brasil. \\ Correspondência \\ M. A. S. Cabrera \\ Departamento de Clínica \\ Médica, Universidade \\ Estadual de Londrina. \\ Rua Montese 65-A, \\ Londrina, $P R$ \\ 86010-015, Brasil. \\ marcoscabrera@uol.com.br
}

\begin{abstract}
This study examines the association between body mass index (BMI), waist-to-hip ratio (WHR), and waist circumference (WC) and allcause and cardiovascular mortality in elderly women in a 5-year longitudinal study of $575 \mathrm{fe}$ male outpatients 60 years and over. The highest $B M I, W H R$, and WC quartiles and predefined $B M I$ categories were analyzed as predictive variables. Death occurred in 88 (15.4\%). Underweight $\left(B M I<18.5 \mathrm{~kg} / \mathrm{m}^{2}\right)$ was associated with all-cause mortality in uni- and multivariate analyses, regardless of age bracket. The survival curves and univariate analysis showed that the highest WHR quartile $(\geq 0.97)$ was associated with all-cause mortality. However, after adjustment for age, smoking, and previous cardiovascular diseases, the increase in WHR was positively associated only in women from 60 to 80 years of age. None of the anthropometric measurements was associated with cardiovascular mortality. The results indicate that underweight and increased waist-to-hip ratio were predictors of all-cause mortality in elderly women, mainly among those under 80 years.
\end{abstract}

Body Mass Index; Obesity; Body Constitution; Mortality
Marcos A. S. Cabrera 1,2

Maurício Wajngarten 2

Otávio C. E. Gebara ${ }^{2}$

Jayme Diament ${ }^{2}$

\section{Introdução}

Muitos estudos na população em geral identificam a obesidade, por meio do índice de massa corporal (IMC), e a distribuição central de gordura corpórea, segundo a relação cintura-quadril (RCQ) e a circunferência abdominal (CA), como fatores de risco para a mortalidade 1 .

Entretanto, essas conclusões não podem ser extrapoladas integralmente para a população idosa, devido a algumas peculiaridades metodológicas relacionadas a esta faixa etária, como o ajuste inadequado das variáveis confundidoras e das doenças preexistentes, a ausência da eliminação da mortalidade precoce, os diferentes tempos de seguimentos e o ajuste da idade no início do estudo 2,3. Além disso, ressalta-se a importância de algumas transformações senescentes como o aumento da gordura corpórea e da diminuição da altura 4 .

Assim, poucos estudos analisaram especificamente os indivíduos acima de 60 anos e dificilmente incluíram idosos com mais de 75 anos. Heiat et al. 5 sugerem que os níveis de IMC relacionados ao aumento de risco de mortalidade em idosos são diferentes dos estabelecidos para a população adulta, e, em algumas análises, a associação de risco é nula ou negativa. De fato, verifica-se que a idade modifica o efeito da obesidade na mortalidade $3,6,7$.

Em relação à obesidade central, poucos autores analisaram a associação da RCQ e da CA 
com a mortalidade em idosos. Por outro lado, apesar de sugerida uma associação maior da RCQ e da CA com a mortalidade, quando comparados aos níveis de IMC, ela foi pouco estudada nas faixas etárias maiores 8,9,10,11,12.

Ademais, ainda não está claro que medida de obesidade central melhor caracteriza o risco de mortalidade em idosos. A circunferência abdominal é mais simples para medir e interpretar e alguns autores demonstraram uma boa associação com a mortalidade 13. Entretanto, outros estudos identificaram a RCQ como melhor preditora de mortalidade, principalmente entre as mulheres 9 .

Neste estudo, nós analisamos mulheres idosas, com idade de 60 a 94 anos, por um período de seguimento de 5 anos e examinamos a associação dos indicadores antropométricos da obesidade central (RCQ e CA) e da obesidade global (IMC) com a mortalidade total e cardiovascular, bem como o impacto de outros importantes fatores de risco nessa associação.

\section{Metodologia}

\section{Delineamento e população estudada}

Estudo de coorte prospectiva, a partir da observação inicial realizada entre junho de 1997 e junho de 1998 e com seguimento até janeiro de 2003 (tempo médio de seguimento de 5,3 anos).

Foram estudadas todas as mulheres com 60 anos ou mais, atendidas em um ambulatório de geriatria de demanda espontânea no período descrito. Foram excluídas 32 pacientes impossibilitadas de permanecer em pé durante a avaliação antropométrica e as portadoras de doença neoplásica ativa, exceto carcinoma basocelular e de células escamosas. Também foram excluídas as pacientes que apresentaram mortalidade precoce - até os primeiros seis meses de seguimento.

\section{Coleta dos dados}

Os dados referentes à identificação, hábitos, doenças existentes, pressão arterial e medidas antropométricas foram obtidos, exclusivamente, por apenas um pesquisador, utilizando-se uma entrevista e uma avaliação clínica. As medidas antropométricas foram realizadas com os indivíduos vestindo roupas leves e sem calçados, na posição ortostática com os pés juntos. Os valores de peso, altura e circunferências foram obtidos com unidades mínimas de 0,1 quilograma, 0,5 centímetro e 0,1 centímetro, respectivamente. A circunferência abdominal foi considerada a menor medida de uma circunferência, no nível da cicatriz umbilical no final do movimento expiratório; e a circunferência do quadril, a maior medida de uma circunferência no nível dos quadris e nádegas 14 .

Foram obtidas amostras sangüíneas, em jejum de 12 horas, para dosagens dos níveis de glicose, colesterol total, lipoproteína colesterol de alta densidade (HDL-c) e triglicerídeos (TG). A lipoproteína colesterol de baixa densidade (LDL-c) foi calculada para indivíduos com triglicerídeos $\leq 400 \mathrm{~g} / \mathrm{dl} 15$.

\section{Variáveis analisadas}

- Variáveis antropométricas: IMC - peso em quilogramas dividido pela altura em metros ao quadrado $\left(\mathrm{kg} / \mathrm{m}^{2}\right)$; CA - em centímetros; RCQ circunferência abdominal dividida pela circunferência do quadril. O IMC, a CA e a RCQ foram analisados como variáveis categóricas estratificadas por quartis. O IMC também foi categorizado pela classificação da Organização Mundial da Saúde: baixo peso (IMC $<18,5 \mathrm{~kg} / \mathrm{m}^{2}$ ), peso normal (IMC $\geq 18,5 \mathrm{e}<25 \mathrm{~kg} / \mathrm{m}^{2}$ ), sobrepeso (IMC $\geq 25 \mathrm{~kg} / \mathrm{m}^{2} \mathrm{e}<30 \mathrm{~kg} / \mathrm{m}^{2}$ ) e obesidade $\left(\mathrm{IMC} \geq 30 \mathrm{~kg} / \mathrm{m}^{2}\right) 1$.

- Idade (anos).

- Doença cardiovascular prévia: foram considerados os indivíduos com diagnóstico clínico e laboratorial de doença coronariana, insuficiência cardíaca e fibrilação atrial durante a avaliação inicial do estudo ou com história referida de infarto agudo do miocárdio ou acidente vascular cerebral prévios.

- Atividade física regular: foram consideradas as mulheres que praticavam algum tipo de atividade física pelo menos três vezes por semana, por no mínimo trinta minutos. Foram excluídos da análise específica dessa variável os indivíduos com atividades restritas exclusivamente ao ambiente domiciliar.

- Baixa renda per capita: foram consideradas as pacientes com renda familiar menor que o percentil 20o correspondente a 1,5 salário mínimo per capita.

- Tabagismo: foram classificadas como fumantes, ex-fumantes e não fumantes.

- Hipertensão arterial: foram consideradas hipertensas as pacientes que referiram o uso de anti-hipertensivos ou apresentaram duas medidas da pressão arterial, em posição deitada, com pressão arterial sistólica acima de $139 \mathrm{mmHg}$ e ou pressão arterial diastólica acima de $89 \mathrm{mmHg} 17$.

- Diabetes melito: mulheres que referiram tratamento farmacológico para diabetes ou apresentaram glicemia de jejum $\geq 126 \mathrm{mg} / \mathrm{dl} 18$. 
- Dislipidemias: os níveis dos lípides analisados foram categorizados utilizando-se os seguintes limites: colesterol total $>200 \mathrm{mg} / \mathrm{dl}$, lipoproteína colesterol de alta densidade (HDLc) $<40 \mathrm{mg} / \mathrm{dl}$, lipoproteína colesterol de baixa densidade (LDL-c) $>130 \mathrm{mg} /$ dl e triglicerídeos $>150 \mathrm{mg} / \mathrm{dl} 18$.

\section{Seguimento e desfechos analisados}

A verificação dos óbitos foi baseada nas seguintes estratégias seqüenciais: retorno em consulta ambulatorial, contato telefônico por assistentes treinados, verificação em prontuários de serviços de saúde e atestado de óbito. Os eventos analisados como desfechos foram a mortalidade total e a mortalidade cardiovascular. Foram considerados como de origem cardiovascular os óbitos cujas causas básicas estão catalogadas na décima revisão da Classificação Internacional das Doenças (CID-10), no capítulo IX, entre os itens I 00 a I 99, correspondentes às doenças cardiovasculares 19, e os óbitos ocorridos devido à morte súbita, caracterizada como a morte que ocorreu até uma hora após o início dos sintomas sem evidência de uma outra causa não cardiovascular 20,21.

\section{Análise estatística}

A análise univariada foi utilizada para determinar o risco relativo (RR) para mortalidade total e cardiovascular, com o intervalo de confiança de 95\%, considerando as seguintes variáveis:

- Variáveis preditivas: os maiores quartis de IMC, RCQ e CA;

- Variáveis confundidoras: diabetes melito, hipertensão arterial, colesterol total $>200 \mathrm{mg} / \mathrm{dl}$, HDL-c < 40mg/dl, LDL-c > 130mg/dl, triglicerídeos $>150 \mathrm{mg} / \mathrm{dl}$, tabagismo, atividade física regular, baixa renda per capita e doenças cardiovasculares prévias.

Os óbitos não cardiovasculares foram caracterizados como censuras para a análise da mortalidade cardiovascular. A análise multivariada foi realizada com os modelos elaborados com as variáveis selecionadas a partir da análise univariada para a mortalidade total, segundo o modelo de riscos proporcionais de Cox e com estratificação para as mulheres com até 80 anos.

Foram obtidas as curvas de sobrevida de Kaplan-Meier, estratificadas por faixa etária, para a mortalidade total de acordo com a classificação de IMC e com os maiores quartis de IMC, RCQ e CA.

O nível de significância utilizado foi de $5 \%$ ( $\alpha=5 \%$ ) e as análises foram realizadas utilizando-se os programas Statistica e SAS Statistical.

\section{Resultados}

Foram estudadas 575 mulheres, de 60 a 94 anos (109 com mais de 80 anos - 18,9\%), com média e mediana de 72,5 e 72,0 anos, respectivamente, em um seguimento médio de 5,3 anos. Cinco idosas foram excluídas do estudo, três por apresentarem câncer e duas devido à morte nos primeiros seis meses de seguimento. Houve perda de seguimento de duas pacientes $(0,3 \%)$, mas somente após o quarto ano de seguimento. Ocorreram $88(15,4 \%)$ óbitos durante o seguimento, sendo 46 (52,3\%) de origem cardiovascular (Tabela 1).

Os valores das variáveis antropométricas que definiram os quartis foram: RCQ $(0,89,0,93$ e 0,97$)$, CA $(85,91$ e $98 \mathrm{~cm})$ e IMC $(22,8,25,8$ e $\left.29,4 \mathrm{~kg} / \mathrm{m}^{2}\right)$.

$\mathrm{Na}$ análise univariada, as idosas com RCQ $\geq$ 0,97 apresentaram maior risco de mortalidade

Tabela 1

Caraterísticas gerais das mulheres estudadas.

\begin{tabular}{|c|c|c|}
\hline Variáveis categóricas & $\mathbf{n}$ & $\%$ \\
\hline Obesidade $\left(\mathrm{IMC} \geq 30 \mathrm{~kg} / \mathrm{m}^{2}\right)$ & 129 & 22,6 \\
\hline Sobrepeso (IMC $\geq 25$ e $\left.<30 \mathrm{~kg} / \mathrm{m}^{2}\right)$ & 189 & 33,2 \\
\hline Normal $\left(I M C \geq 18,5\right.$ e $\left.<25 \mathrm{~kg} / \mathrm{m}^{2}\right)$ & 225 & 39,5 \\
\hline Baixo peso $\left(\mathrm{IMC}<18,5 \mathrm{~kg} / \mathrm{m}^{2}\right)$ & 27 & 4,7 \\
\hline Hipertensão arterial & 321 & 56,0 \\
\hline Diabetes mellitus & 122 & 14,6 \\
\hline Doença cardiovascular prévia & 93 & 16,3 \\
\hline Tabagismo & 28 & 4,9 \\
\hline Tabagismo e ex-tabagismo & 106 & 18,4 \\
\hline Atividade física regular & 121 & 21,1 \\
\hline Baixa renda per capita (< percentil $\left.20^{\circ}\right)$ & 91 & 15,9 \\
\hline Variáveis numéricas & Média & $\begin{array}{l}\text { Desvio } \\
\text { padrão }\end{array}$ \\
\hline Idade (anos) & 72,5 & 8,0 \\
\hline $\mathrm{IMC}\left(\mathrm{kg} / \mathrm{m}^{2}\right)$ & 26,3 & 4,9 \\
\hline Relação cintura-quadril & 0,92 & 0,06 \\
\hline Circunferência abdominal (cm) & 91,2 & 10,6 \\
\hline Colesterol total (mg/dl) & 210,6 & 43,3 \\
\hline $\mathrm{HDL}-\mathrm{c}(\mathrm{mg} / \mathrm{dl})$ & 52,4 & 14,2 \\
\hline LDL-c (mg/dl) & 128,5 & 38,3 \\
\hline Triglicerídeos (mg/dl) & 148,7 & 82,5 \\
\hline Mortalidade & $\mathbf{n}$ & $\%$ \\
\hline Mortalidade total & 88 & 15,4 \\
\hline Mortalidade cardiovascular & 46 & 8,1 \\
\hline
\end{tabular}

IMC = índice de massa corporal; HDL-c = lipoproteína colesterol de alta densidade; LDL-c = lipoproteína colesterol de baixa densidade. 
total ( $\mathrm{p}<0,05)$, o que não aconteceu com as de maiores níveis de CA e IMC. Os maiores quartis de RCQ, CA e IMC não apresentaram aumento significativo do risco para mortalidade cardiovascular. A obesidade e o sobrepeso não estavam associados ao maior risco de mortalidade total e cardiovascular. Entretanto, as idosas com baixo peso apresentaram uma forte associação com mortalidade total ( $\mathrm{p}<0,001)$ (Tabela 2).

As covariáveis associadas com maior risco de mortalidade total e cardiovascular foram: diabetes, tabagismo, ausência de atividade física regular e a doença cardiovascular prévia. As idosas com níveis reduzidos de HDL-c apresentaram uma associação positiva somente com a mortalidade total (Tabela 2).

No modelo de análise multivariada, o mais alto quartil de RCQ não estava associado à mortalidade total, independentemente de idade, tabagismo, doença cardíaca prévia e diabetes. Entretanto, quando se excluíam as mulheres com mais de 80 anos, o aumento da RCQ apresentava um risco para mortalidade inde- pendente das variáveis analisadas. O baixo peso apresentou-se associado de maneira independente à mortalidade, mesmo sem a estratificação etária (Tabela 3).

As curvas de sobrevida de Kaplan-Meier mostraram uma mortalidade maior das idosas com baixo peso e um comportamento semelhante entre aquelas com peso normal e as com sobrepeso (Figura 1). Além disso, ratificaram o maior risco de mortalidade total das mulheres com maiores níveis de RCQ ( $p=0,02$ ) (Figura 2).

\section{Discussão}

Embora a obesidade seja um fator de risco para a mortalidade e eventos cardiovasculares na população em geral, esta associação é pouco estudada entre os idosos, particularmente em nosso meio 22 .

A média de idade (72,5 anos) das mulheres estudadas foi alta, quando comparada com a de outros autores, que freqüentemente incluem

Tabela 2

Análise univariada dos fatores associados à mortalidade total e cardiovascular.

\begin{tabular}{|c|c|c|}
\hline Variáveis & $\begin{array}{l}\text { Mortalidade total } \\
\text { RR (IC95\%) }\end{array}$ & $\begin{array}{c}\text { Mortalidade cardiovascular } \\
\text { RR (IC95\%) }\end{array}$ \\
\hline Idade (anos) & $1,11(1,09-1,12)^{\star}$ & $1,10(1,08-1,11)^{\star}$ \\
\hline Obesidade (IMC $\left.\geq 30 \mathrm{~kg} / \mathrm{m}^{2}\right)$ & $0,65(0,38-1,11)$ & $0,61(0,28-1,33)$ \\
\hline Obesidade (IMC $\left.\geq 30 \mathrm{~kg} / \mathrm{m}^{2}\right) \#$ & $0,71(0,41-1,23)$ & $0,62(0,28-1,34)$ \\
\hline Sobrepeso $\left(I M C \geq 25\right.$ and $\left.<30 \mathrm{~kg} / \mathrm{m}^{2}\right) \#$ & $0,98(0,62-1,55)$ & $0,84(0,45-1,57)$ \\
\hline Baixo peso (IMC $\left.<18,5 \mathrm{~kg} / \mathrm{m}^{2}\right) \#$ & $2,86(1,75-4,76)^{\star}$ & $1,45(0,48-4,39)$ \\
\hline IMC - 4o quartil ( $\left.\geq 29,4 \mathrm{~kg} / \mathrm{m}^{2}\right) \# \#$ & $0,63(0,37-1,06)$ & $0,64(0,31-1,34)$ \\
\hline RCQ - 4o quartil $(\geq 0,97) \# \#$ & $1,56(1,05-2,32)^{\star \star}$ & $1,31(0,73-2,38)$ \\
\hline CA - 4ㅇ quartil ( $\geq 98 \mathrm{~cm}) \# \#$ & $0,98(0,63-1,52)$ & $1,03(0,55-1,93)$ \\
\hline Hipertensão arterial & $0,97(0,66-1,42)$ & $1,78(0,97-3,03)$ \\
\hline Diabetes melito & $1,89(1,25-2,94)^{\star \star \star}$ & $2,27(1,25-4,17)^{\star \star \star}$ \\
\hline Colesterol total > 200mg/dl & $0,63(0,43-0,93)^{\star \star}$ & $0,95(0,54-1,67)$ \\
\hline $\mathrm{HDL}-\mathrm{c}<40 \mathrm{mg} / \mathrm{dl}$ & $1,64(1,10-2,43)^{\star \star \star}$ & $1,51(0,84-2,70)$ \\
\hline $\mathrm{LDL}-\mathrm{c}>130 \mathrm{mg} / \mathrm{dl}$ & $0,57(0,37-0,86)^{\star \star \star}$ & $0,71(0,40-1,26)$ \\
\hline Triglicerídeos > 150mg/dl & $1,07(0,72-1,61)$ & $1,00(0,56-1,75)$ \\
\hline Tabagismo e ex-tabagismo & $1,67(1,09-2,50)^{\star \star \star}$ & $2,12(1,19-3,84)^{\star \star \star}$ \\
\hline Atividade física regular\#\#\# & $0,44(0,20-0,93)^{\star \star}$ & $0,11(0,02-0,82)^{\star \star \star}$ \\
\hline Doença cardiovascular prévia & $3,22(2,22-4,76)^{\star}$ & $5,55(3,34-9,99)^{\star}$ \\
\hline Baixa renda per capita (< percentil $\left.20^{\circ}\right)$ & $1,28(0,80-2,04)$ & $1,29(0,65-2,63)$ \\
\hline
\end{tabular}

$\mathrm{RCQ}=$ relação cintura-quadril; IMC = índice de massa corporal; CA = circunferência abdominal;

$\mathrm{RR}=$ risco relativo; $I \mathrm{C}=$ intervalo de confiança; $\mathrm{HDL}-\mathrm{C}=$ lipoproteína colesterol de alta densidade;

$\mathrm{LDL}-\mathrm{C}=$ lipoproteína colesterol de baixa densidade.

${ }^{*} \mathrm{p}<0,001$.

$\star \star p<0,05$

$\star \star \star ~ p<0,01$.

\# Comparado com IMC $\geq 18,5$ e $<25 \mathrm{~kg} / \mathrm{m}^{2}$

\#\# Comparado com os demais quartis (1으, 2으 e 3 의)

\#\#\# Excluídos os pacientes com atividades exclusivamente intradomiciliares. 
Análise multivariada dos fatores associados à mortalidade total.

\begin{tabular}{|c|c|c|c|c|c|c|}
\hline \multirow[t]{2}{*}{ Variáveis } & \multicolumn{3}{|c|}{ Todas as idosas } & \multicolumn{3}{|c|}{$60-80$ anos } \\
\hline & $\beta(E P)$ & $\mathrm{RR}$ & $\mathrm{p}$ & $\beta(E P)$ & $\mathrm{RR}$ & $p$ \\
\hline \multicolumn{7}{|l|}{ Modelo 1} \\
\hline $\mathrm{RCQ} \geq 0,97$ & $0,30(0,23)$ & 1,35 & 0,18 & $0,66(0,32)$ & 1,94 & 0,03 \\
\hline Doença cardiovascular prévia & $0,78(0,24)$ & 2,18 & 0,001 & $0,99(0,32)$ & 2,29 & 0,01 \\
\hline Tabagismo e ex-tabagismo & $0,84(0,25)$ & 2,33 & $<0,001$ & $1,32(0,32)$ & 3,75 & $<0,001$ \\
\hline Diabetes melito & $0,48(0,26)$ & 1,62 & 0,06 & $0,83(0,32)$ & 2,29 & 0,01 \\
\hline Idade (anos) & $0,09(0,01)$ & 1,10 & $<0,001$ & $0,12(0,03)$ & 1,12 & $<0,001$ \\
\hline \multicolumn{7}{|l|}{ Modelo 2} \\
\hline Baixo peso $\mid \mathrm{MC}<18,4 \mathrm{~kg} / \mathrm{m}^{2}$ & $0,75(0,36)$ & 2,12 & 0,03 & $1,58(0,50)$ & 4,86 & 0,001 \\
\hline Doença cardiovascular prévia & $0,72(0,23)$ & 2,05 & 0,002 & $0,83(0,32)$ & 2,30 & 0,009 \\
\hline Tabagismo e ex-tabagismo & $0,83(0,25)$ & 2,30 & $<0,001$ & $1,24(0,50)$ & 3,46 & $<0,001$ \\
\hline Diabetes melito & $0,57(0,26)$ & 1,77 & 0,03 & $1,10(0,32)$ & 3,01 & $<0,001$ \\
\hline Idade (anos) & $0,09(0,01)$ & 1,10 & $<0,001$ & $0,12(0,02)$ & 1,12 & $<0,001$ \\
\hline
\end{tabular}

$\mathrm{RCQ}=$ relação cintura-quadril; IMC = índice de massa corporal; $\mathrm{RR}=$ risco relativo; $\mathrm{EP}=$ erro padrão.

indivíduos com até 70 ou 75 anos 8,9,10,11. Neste estudo, 228 mulheres tinham 75 anos ou mais $(40,0 \%)$

A análise dos dados e a extrapolação das conclusões devem considerar as características da população estudada, restrita a uma clientela de idosos de ambulatórios de geriatria com demanda espontânea e abrangendo diferentes níveis sociais. Diferentemente da maioria dos estudos realizados, em que os próprios pacientes realizavam as medidas antropométricas 5 , essas medidas e a coleta dos dados foram realizadas apenas por um pesquisador, favorecendo a homogeneidade dos resultados.

A exclusão dos idosos com diagnóstico de câncer e da mortalidade precoce contribuiu para diminuir a interferência das possíveis comorbidades nos índices de mortalidade. Além disso, a identificação das doenças cardiovasculares preexistentes possibilitou uma análise diferenciada da influência dessa importante variável confundidora.

Os resultados mostraram que a obesidade central, representada pelo aumento da RCQ, e não a obesidade global, representada pelo aumento de IMC, foi preditora de mortalidade total em idosas, principalmente as com até 80 anos. Estes dados foram confirmados por outros autores que estudaram mulheres idosas $8,9,11$. No entanto, Visscher et al. 10 , em um seguimento de 4 a 5 anos, não encontraram nenhuma associação estatisticamente significativa entre medidas antropométricas e mortalidade em idosos. Em tempo de seguimento me-
Figura 1

Curvas de sobrevida de Kaplan-Meier para mortalidade total de acordo com a classificação do índice de massa corporal (IMC).

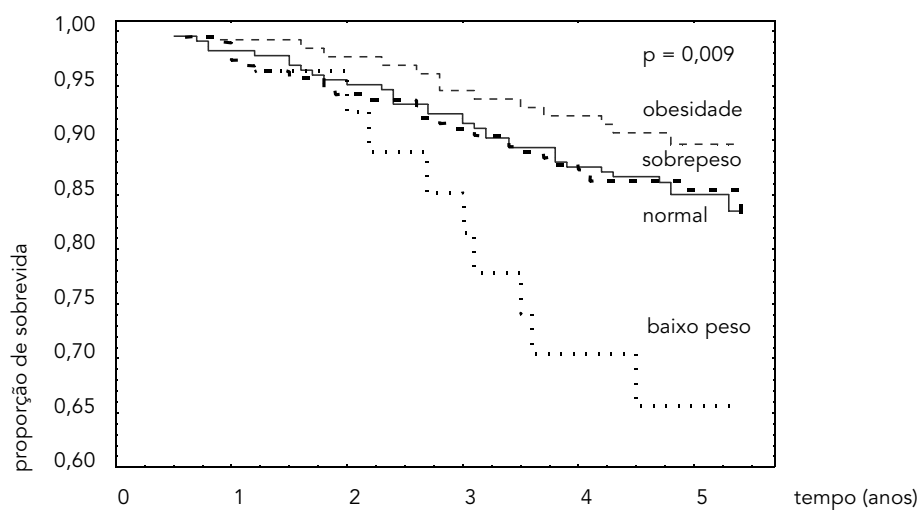

nor ( 3 anos), Woo et al. 13 estudaram idosos com 70 anos ou mais e não encontraram uma associação positiva entre RCQ e mortalidade, apesar da associação negativa obtida pelo IMC 12.

Em nosso estudo, a RCQ associou-se à mortalidade total na análise multivariada, porém apenas quando se excluíam as mulheres com mais de 80 anos. Como a população estudada incluía idosas com até 94 anos, a idade, por si 
Figura 2

Curvas de sobrevida de Kaplan-Meier para mortalidade total de acordo com os maiores quartis de relação cintura-quadril, circunferência abdominal e índice de massa corporal.

Figura 2a

Relação cintura-quadril (RCQ).
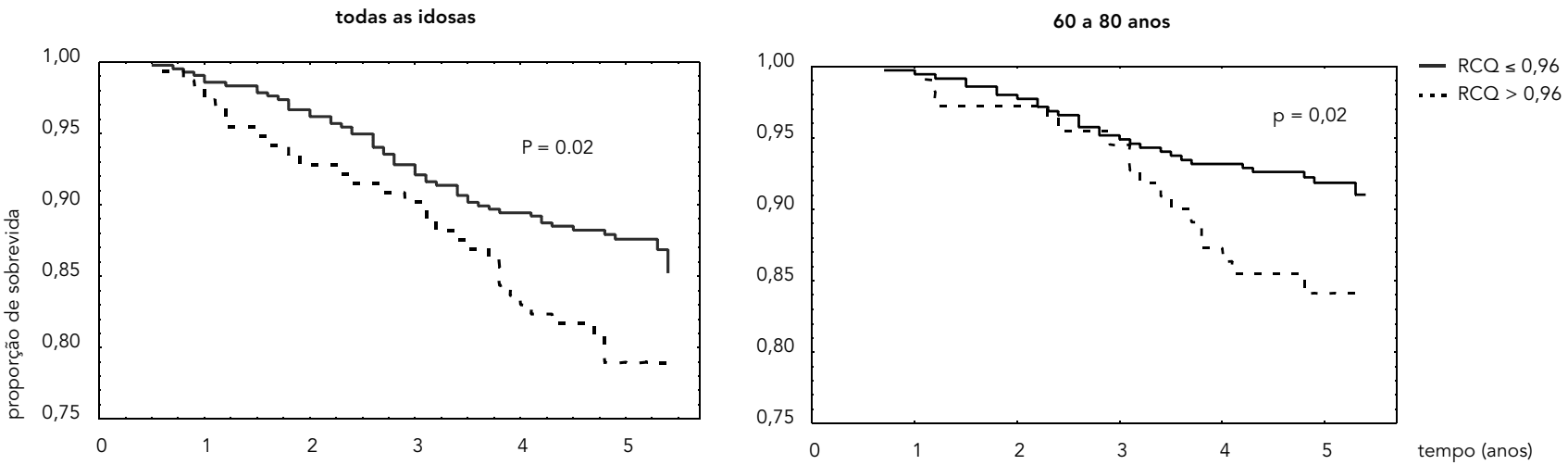

Figura $2 b$

Circunferência abdominal (CA).
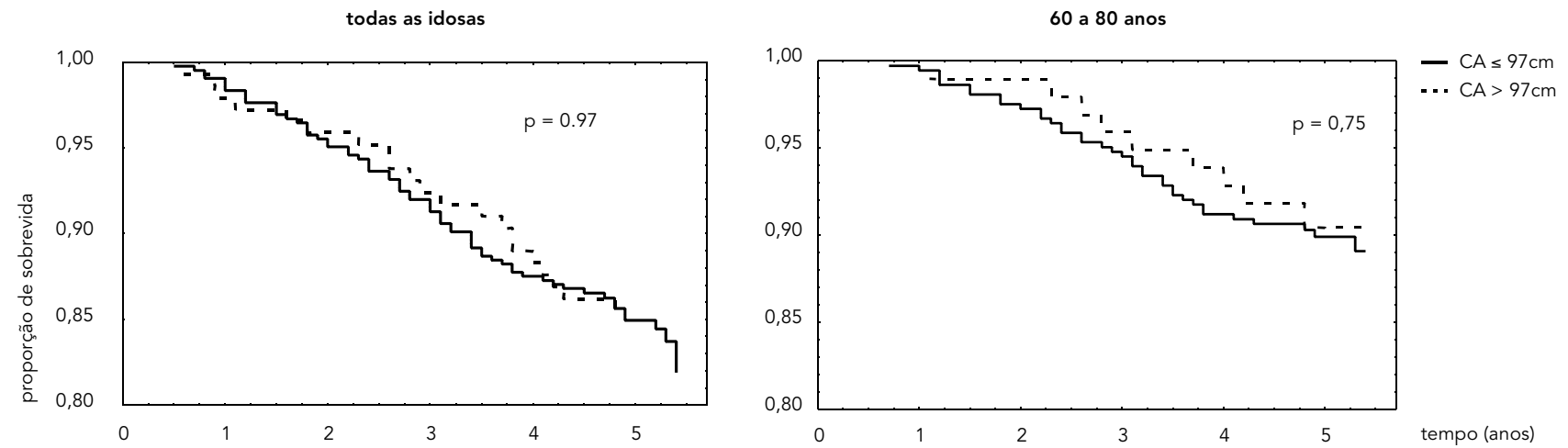

Figura 2c

Índice de massa corporal (IMC).
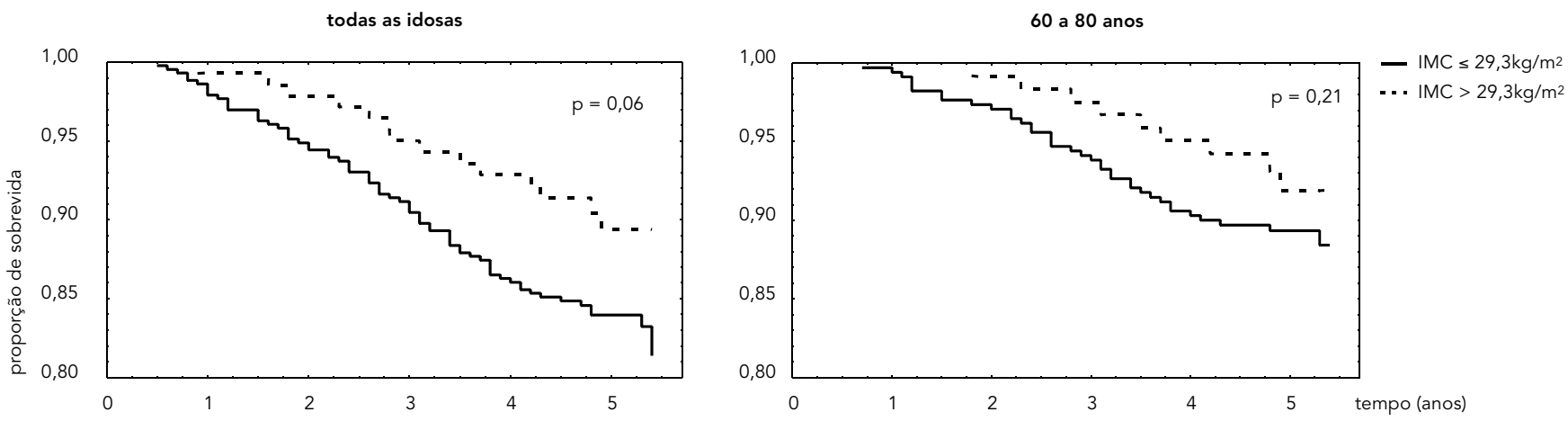
só, representava um grande fator de risco para mortalidade nessa faixa etária excluída na referida análise.

Em contraste aos resultados obtidos com a RCQ, não observamos uma associação entre a CA e a mortalidade. Embora a CA seja uma importante medida na avaliação da gordura abdominal na população em geral 1, entre os idosos a CA foi relacionada mais com gordura total do que com a abdominal 23 . Isso poderia explicar por que a CA é raramente associada à mortalidade em idosos 13 .

Observamos que as pacientes obesas e com sobrepeso (IMC $\geq 25 \mathrm{~kg} / \mathrm{m}^{2}$ ) não apresentaram aumento do risco da mortalidade como foi observado em população mais jovem ${ }^{1}$. Muitos autores têm estudado a associação do IMC e mortalidade nesta faixa etária e os resultados são conflitantes. Os estudos com maiores períodos de seguimento apresentam predomínio de associação positiva 24,25,26, enquanto, em outros, a associação encontrada é negativa 12,27 ou nula $9,11,28,29,30,31,32,33,34$.

Em concordância aos resultados obtidos, os estudos com tempos de seguimento semelhantes ao apresentado (em torno de 5 a 6 anos) também encontraram associações nulas ou negativas 9,11,28,29,34. Entretanto, Cornoni-Huntley et al. 35 observaram uma associação positiva entre níveis altos de IMC (percentil 85o) e mortalidade em idosos de 65 a 74 anos. Porém, nesse estudo o tempo de seguimento foi variável e de, no mínimo, 7 anos. Em estudo que analisou idosos com 70 anos ou mais por 6 anos, observou-se um aumento de mortalidade em mulheres, mas apenas a partir de níveis muito altos de IMC (> 35kg/m²) 28 .

Os dados confirmam o baixo peso como um importante fator de risco para a mortalidade total, mesmo quando comparado apenas aos indivíduos com o IMC entre 18,5 e $24,9 \mathrm{~kg} / \mathrm{m}^{2}$. Provavelmente, o aumento da mortalidade com menores IMC está no fato de o IMC não ser o melhor marcador de adiposidade, pois ele reflete a quantidade de massa gorda e também de massa magra, e ambas têm interferências distintas na saúde e na longevidade 36 . Assim, a associação em forma de curva U entre IMC e mortalidade em idosos parece ser conseqüência da composição das funções de risco lineares e opostas das porcentagens de gordura corpórea e da massa magra com a mortalidade.

Assim, a medida antropométrica de composição de gordura corpórea, como a RCQ, pode representar um melhor parâmetro de massa gorda que o IMC em idosos e parece refletir melhor o papel deletério do aumento de gordura na mortalidade dessa população.
Em relação à mortalidade cardiovascular, observou-se que a obesidade, o sobrepeso, os maiores quartis de IMC, RCQ e CA não apresentaram uma associação positiva, estatisticamente significante. Assim, com os dados apresentados, os resultados de outros estudos com mortalidade cardiovascular e IMC são menos conclusivos que os com mortalidade total. Foram relatadas associações nulas, negativas $27 \mathrm{e}$ positivas 37 , mas, quando positiva, essa associação não é forte e não persiste após os 75 anos 25. Apesar disso, a associação da obesidade com os fatores de riscos cardiovasculares não desaparece com a idade e persiste mesmo após os 75 anos 37,38 .

Poucos autores estudaram a associação de RCQ e CA e doença cardiovascular em idosas. Apesar de os nossos resultados não evidenciarem essa associação significativa, dados obtidos pelo Iowa Women's Health Study em mulheres de 55 a 69 anos mostraram que a RCQ foi identificada também como preditora, de mortalidade cardiovascular 10,39. A CA foi identificada como forte preditora de doença coronariana em mulheres de 40 a 59 anos do Nurses Health Study, porém, entre mulheres de 60 a 65 anos, essa associação era mais fraca 7 .

\section{Limitações do estudo}

Este estudo apresenta algumas limitações referentes à seleção da amostra, que incluiu somente idosas ambulatoriais, e ao número de idosas estudadas, o que poderia restringir a força para a identificação do papel preditivo de algumas variáveis na análise do risco de morte.

A análise da mortalidade cardiovascular pode ter sido influenciada pelo elevado número de censuras, representado pelas mortes de origem não cardiovascular. Além disso, a variabilidade e a heterogeneidade presentes no processo de envelhecimento interferem na possibilidade de generalizar estas conclusões para as mulheres idosas em geral.

Entretanto, apesar disto, nossos resultados permitem algumas conclusões, pois as idosas estudadas apresentam características representativas da maioria das mulheres desta faixa etária.

\section{Conclusões}

Os dados apresentados demonstram que, neste grupo de idosas estudadas, a obesidade e o sobrepeso não apresentaram aumento de risco de mortalidade, enquanto o baixo peso se confirmou como um importante determinante de mortalidade. 
Alem disso, os resultados ressaltam a importância da aferição da RCQ como parâmetro antropométrico de distribuição de gordura central na análise de risco entre as idosas, cujo o aumento demonstrou ser um fator de risco para a mortalidade total.

\section{Resumo}

Este estudo analisa a associação entre a relação cintura-quadril (RCQ), a circunferência abdominal (CA) e o indice de massa corporal (IMC) com a mortalidade total e cardiovascular em 575 mulheres idosas ambulatoriais por um seguimento de cinco anos. Os maiores quartis de RCQ, CA e IMC, bem como as categorias pré-determinadas de IMC, foram analisados como variáveis preditivas e analisada a interferência de algumas variáveis confundidoras. Oitenta e oito mulheres morreram durante o seguimento $(15,4 \%)$. As mulheres com baixo peso (IMC $\left.<18,5 \mathrm{~kg} / \mathrm{m}^{2}\right)$ apresentavam uma associação positiva com a mortalidade total nas análises uni e multivariadas, independentemente da estratificação etária. Nas curvas de sobrevida e na análise univariada, o maior quartil de RCQ $(\geq 0,97)$ estava associado com a maior mortalidade total, entretanto, na análise multivariada o aumento de RCQ apresentou uma associação independente com a mortalidade total, apenas entre as mulheres de 60 a 80 anos. Nenhuma medida antropométrica apresentou uma associação significativa com a mortalidade cardiovascular. Os resultados identificaram o baixo peso e a $R C Q$ como preditores de mortalidade total em idosas, principalmente entre as mulheres com até 80 anos.

Índice de Massa Corporal; Obesidade; Constituição Corporal; Mortalidade

\section{Colaboradores}

M. A. S. Cabrera contribuiu na elaboração do projeto, levantamento bibliográfico, coleta de dados, análise dos resultados, análise estatística e elaboração do artigo. M. Wajngarten colaborou na elaboração do projeto, análise dos resultados e elaboração do artigo. O. C. E. Gebara participou do levantamento bibliográfico, análise dos resultados, análise estatística e elaboração do artigo. J. Diament contribui na análise dos resultados, elaboração do artigo e revisão ortográfica.

\section{Agradecimentos}

Agradecemos a colaboração do Prof. Dr. Rui Laurenti e do Prof. Dr. José Maria de Souza, da Faculdade de Saúde Pública, Universidade de São Paulo e também da Prof. Dra. Tiemi Matsuo, do Departamento de Matemática, Universidade Estadual de Londrina.

\section{Referências}

1. World Health Organization. Obesity: preventing and manging the global epidemic - report of a WHO consultation on obesity. Geneva: World Health Organization; 2000.

2. Kushner RF. Body weight and mortality. Nutr Rev 1993; 51:127-36.

3. Stevens J. Impact of age on association between weight and mortality. Nutr Rev 2000; 58:129-37.

4. Baumgartner RN. Body composition in healthy aging. Ann N Y Acad Sci 2000; 904:437-48.

5. Heait A, Vaccarino V, Krumholz HM. An evidencebased assessment of federal guidelines for overweight and obesity as they apply to elderly persons. Arch Intern Med 2001; 161:1194-203.

6. Manson JE, Willett WC, Stampfer MJ. Body weight and mortality among women. N Engl J Med 1995; 333:677-85.

7. Rexrode KM, Carey VJ, Hennekens CH, Walters EE, Colditz GA, Stampfer MJ, et al. Abdominal adiposity and coronary heart disease in women. JAMA 1998; 280:1843-8

8. Folsom AR, Kaye AS, Sellers TA, Hong C-P, Cerhan JR, Potter JD, et al. Body fat distribution and 5year risk of death in older women. JAMA 1993; 269:483-7.

9. Folsom AR, Kushi LH, Anderson KE, Mink PJ, Olson JE, Hong CP, et al. Associations of general and abdominal obesity with multiple health outcomes in older women: the Iowa Women's Health Study. Arch Intern Med 2000; 160:2117-28.

10. Visscher TLS, Seidel JC, Molarius A, van der Kuip D, Hofman A, Witteman JCM. A comparison of body mass index, waist-hip ratio and waist circunference as predictors of all-cause mortality among the elderly: the Rotterdam study. Int J Obes 2001; 25:1730-5.

11. Lahmann PH, Lissner L, Gullberg B, Berglund G. A prospective study of adiposity and all-cause mortality: The Malmo Diet and Cancer Study. Obes Res 2002; 10:361-9.

12. Woo J, Ho SC, Sham A. Longitudinal changes in body mass index and body composition over 3 years and relationship to health outcomes in Hong Kong chinese age 70 and older. J Am Geriatr Soc 2001; 49:737-46.

13. Woo J, Ho SC, Yu AL, Sham A. Is waist circumference a useful measure in predicting health outcomes in the elderly? Int J Obes Relat Metab Disord 2002; 26:1349-55.

14. Kissebah AH, Krakower GR. Regional adiposity and mortality. Physiol Rev 1994; 74:761-811. 
15. Friedewald WT, Levy RI, Fredrickson DS. Estimation of the concentration of low-density lipoprotein cholesterol in plasma, without use of the preparative ultracentrifuge. Clin Chem 1972; 18: 499-502.

16. Mion Jr. D, Gomes MAM, Nobre F, Amodeo C, Kohlmann Jr. O, Praxedes JN, et al. IV diretrizes brasileiras de hipertensão arterial. Arq Bras Cardiol 2004; 82 Suppl 4:7-14.

17. Anonymous. Report of the Expert Committee on the Diagnosis and Classification of Diabetes Mellitus. Diabetes Care 1997; 20:1183-97.

18. Santos, RD. III diretrizes brasileiras sobre dislipidemias e diretriz de prevenção de arteriosclerose. Arq Bras Cardiol 2001; 77 Suppl 3:1-48.

19. Organização Mundial da Saúde. Classificação Estatística Internacional de Doenças e Problemas Relacionados à Saúde, 10a revisão. v. 3. São Paulo: Centro Colaborador da OMS para a Classificação de Doenças em Português; 1998.

20. Gordon T, Kannel WB. Premature mortality from coronary heart disease. The Framingham Study. JAMA 1971; 215:1617-25.

21. Mäkikallio TH, Huikuri HV, Mäkikallio A, Sourander LB, Mitrani RD, Castellanos A, et al. Prediction of sudden cardiac death by fractal analysis of heart rate variability in elderly subjects. J Am Coll Cardiol 2001; 37:1395-402.

22. Marafon LP, Cruz IB, Schwanke CH, Moriguchi EH. Preditores cardiovasculares da mortalidade em idosos longevos. Cad Saúde Pública 2003; 19:799-807.

23. Harris TB, Visser M, Everhart J, Cauley J, Tylavsky F, Fuerst T, et al. Waist circumference and sagittal diameter reflect total body fat better than visceral fat in older men and women. The Health, Aging and Body Composition Study. Ann N Y Acad Sci 2000; 904:462-73.

24. Lindsted KD, Singh PN. Body mass and 26-year risk of mortality among women who never smoked: findings from the Adventist Mortality Study. Am J Epidemiol 1997; 146:1-11.

25. Calle EE, Thun MJ, Petrelli JM, Rodriguez C, Heath JR. Body-mass index and mortality in a prospective cohort of U.S. adults. N Engl J Med 1999; 341:1097-105.

26. Stevens J, Cai J, Pamuk ER, Williamson DF, Thun MJ, Wood JL. The effect of age on the association between body-mass index and mortality. N Engl J Med 1998; 338:1-7.

27. Grabowski DC, Ellis JE. High body mass index does not predict mortality in older people: analysis of the Longitudinal Study of Aging. J Am Geriatr Soc 2001; 49:868-979.
28. Allison DB, Gallagher D, Heo M, Pi-Sunyer FX, Heymsfield SB. Body mass index and all-cause mortality among people age 70 and over: the Longitudinal Study of Aging. Int J Obes Relat Metab Disord 1997; 21:424-31.

29. Diehr P, Bild DE, Harris TB, Duxbury A, Siscovick D, Rossi M. Body mass index and mortality in nonsmoking older adults: The Cardiovascular Health Study. Am J Public Health 1998; 88:623-9.

30. Somes GW, Kritchevsky SB, Shorr RI, Pahor M, Applegate WB. Body mass index, weight change, and death in older adults. The Systolic Hypertension in the Elderly Program. Am J Epidemiol 2002; 156:132-8.

31. Fried LP, Kronmal RA, Newman AB, Bild DE, Mittelmark MB, Polak JF, et al. Risk factors for 5-year mortality in older adults. The Cardiovascular Health Study. JAMA 1998; 279:585-92.

32. Rissanen A, Knekt P, Heliovaara M, Aromaa A, Reunanen A, Maatela J. Weight and mortality in finnish women. J Clin Epidemiol 1991; 44:787-95.

33. Tayback M, Kumanyika S, Chee E. Body weight as a risk factor in the elderly. Arch Intern Med 1990; 150:1065-72.

34. Landi F, Zuccalá G, Gambassi G, Incalzi RA, Manigrasso L, Pagano F, et al. Body mass index and mortality among older people living in the community. J Am Geriatr Soc 1999; 47:1072-6.

35. Cornoni-Huntley JC, Harris TB, Everett DF, Albanes D, Micozzi MS, Miles TP, et al. An overview of body weight of older persons, including the impact of mortality. J Clin Epidemiol 1991; 44:74353.

36. Allison DB, Faith MS, Heo M, Kotler DP. Hypothesis concerning the $U$-shaped relation between body mass index and mortality. Am J Epidemiol 1997;146:339-49.

37. Rimm EB, Stampfer MJ, Giovannucci E, Ascherio A, Spiegelman D, Colditz GA, et al. Body size and fat distribution as predictors of coronary heart disease among middle-aged and older US men. Am J Epidemiol 1995; 141:1117-27.

38. Cabrera MAS, Jacob Filho W. Obesity in elderly: prevalence, distribution and association with habits and comorbidities. Arq Bras Endocrinol Metabol 2001; 45:494-501.

39. Prineas RJ, Folsom AR, Kaye AS. Central adiposity and increased risk of coronary artery disease mortality in older women. Ann Epidemiol 1993; 3:3541 .

Recebido em 04/Mar/2004

Versão final reapresentada em 10/Nov/2004 Aprovado em 07/Dez/2004 